\title{
EFFECT OF ANODE SHAPE AND ELECTROLYTE ON THE EFFICIENCY CHARACTERISTICS OF SACRIFICE ANODE IMMERSE METHOD WITH DNVGL-RPB401
}

\author{
TUBAGUS NOOR ROHMANNUDIN* \\ Department of Materials and Metallurgical Engineering \\ Institut Teknologi Sepuluh Nopember \\ Jl. Teknik Kimia, Keputih, 60111, Surabaya,East Java, Indonesia \\ roma_r2001@yahoo.com \\ ZAID SULAIMAN \\ Department of Materials and Metallurgical Engineering \\ Institut Teknologi Sepuluh Nopember \\ Jl. Teknik Kimia, Keputih, 60111, Surabaya,East Java, Indonesia \\ sulaimanzaid97@gmail.com \\ LUKMAN NOEROCHIM \\ Department of Materials and Metallurgical Engineering \\ Institut Teknologi Sepuluh Nopember \\ Jl. Teknik Kimia, Keputih, 60111, Surabaya,East Java, Indonesia \\ lukman@mat-eng.its.ac.id \\ * Corresponding author
}

Published 31 January 2020

\begin{abstract}
Corrosion is a damage which occurs naturally. One method of corrosion prevention is cathodic protection using the sacrificial anoda. one standard commonly used in cathodic protection design is DnVGL-RPB401. In addition, DnVGLRPB401 method can be used to measure anoda current capacity. In this research, the variation of shape of the anode was the shape of a cylinder anode, beam and cone. The variation of electrolyte is pure $\mathrm{NaCl}$ solution, Kitchen Salt solution and Kenjeran sea water. In the electrolyte pure $\mathrm{NaCl}$ solution, each anode form has an efficiency of $86 \%, 83 \%$, and $78 \%$ respectively. the cylindrical anode shape has the highest efficiency because the same distance between anode and cathode at the cylindrical anode.so that the current distribution is evenly distributed. Then in the form of cylindrical anodes, each electrolyte medium has an efficiency of $114 \%, 86 \%$ and $83 \%$ respectively. The salt solution has the highest efficiency because of the low content of $\mathrm{NaCl}$ which is the cause of corrosion and the presence of potassium Iodide which is a corrosion inhibitor.
\end{abstract}

Keywords: DNVGL-RPB401; electrochemical; electrolyte; anode shape; anode efficiency

\section{Introduction}

One of the cathodic protection is the sacrificial anode. Anodes are made of more "active" potential (more negative potential) metal alloys than protected structures (cathodes). The potential difference between the two metals means that the sacrificial anode material corrodes in preference to structure. This effectively stops the oxidation reaction in the protected metal (Pierre, 2008)

Zinc metal and aluminum were generally used in salt water where resistivity was generally lower. Its was used for hulls, boats and offshore pipelines. One of the most common sacrificial anode method standards in the industry was the DnVGL-RPB401 standard. The DnVGL-RPB401 (2017) method standard is used to determine the anode consumption rate, anode efficiency, anode potential and anode current capacity . In the DnVGLRPB401 method, the anode is cylindrical. While the fact was that not a few sacrificial anodes were used besides cylinders. 
MATERIALS RESEARCH COMMUNICATIONS 1(1) (2020) 8-16

Tubagus et. al.

Galvanic Anode Modeling Cathodic Protection System with Dynamic Polarization characteristics, that the difference in distance between the anode and the cathode affects the electrical potential and current capacity at the sacrificial anode. On the surface of the anode, the anode got closer to the cathode. then the value of the electric potential is more positive, and the value of the current is higher. The distribution of the protection current density is inversely proportional to the surface of the galvanic anode. This is because the galvanic anode close to the cathode will dissolve faster (Mujezinovic, 2016 ; Turkovic, 2017)

Among all anions, the chloride ion was the ion which has the highest concentration of power in the oxide layer, this is due to their small size and the ease with which the chloride ion moves. then the chloride ion causes a decrease in the oxide layer. In addition, the depth of corrosion of holes in the metal also increases with the concentration of chloride ions (Vargel, 2004). Compared to chloride ions, sulfate ions do have a lower corrosion attack on metals. However, the presence of sulfate ions can increase the depth of corrosion in the metal (Vargel, 2004). The presence of potassium iodide in solution, causing ionic iodide tends to oxidize to Tri-Iodide $\left(\mathrm{I}_{3}{ }^{-}\right)$, this negative charge will absorb on metals whose layers have a positive charge. So that the iodide ion $\left(\mathrm{I}^{-}\right)$will form a layer on the metal surface and will inhibit corrosion on the metal (Khadom, 2018)

The Arrhenius Theory explains that the conductivity in electrolyte solutions is determined by electrolyte ions. The conductivity of a media illustrates the media's ability to carry electricity. In a liquid composed of many ions, it will easily transfer electricity. If there is more $\mathrm{Na}+$ and $\mathrm{Cl}$ - then more electricity is carried or transferred and this means higher conductivity of the liquid. The results of the conductivity measurement of $\mathrm{NaCl}$ solution at various percentages, show that there is a linear relationship between the conductivity value and the percentage of $\mathrm{NaCl}$. The higher the concentration, the greater the conductivity value. So that will make the electrical resistance in $\mathrm{NaCl}$ solution will decrease with increasing $\mathrm{NaCl}$ concentration (Widodo, 2018). on Aluminum metal, Potassium Iodate causes an increase in the oxide layer before soaking (el-Maghraby, 2009).

\section{Experimental}

\subsection{Spesification of Sacrificial Anode}

In Table 1 was the composition of the Aluminum anode that will be used as a sacrificial anode

Table 1. Composition of the Aluminum anode

\begin{tabular}{cc}
\hline \hline Element & Composition (\%) \\
\hline $\mathrm{Al}$ & 95.81 \\
$\mathrm{Zn}$ & 2.78 \\
$\mathrm{Fe}$ & 0.7 \\
$\mathrm{Si}$ & 0.44 \\
$\mathrm{Cu}$ & 0.12 \\
$\mathrm{~Pb}$ & 0.01 \\
\hline \hline
\end{tabular}

\subsection{Cutting of sacrificial anode}

Anode specimens were grinded in 3 shapes that have the same surface area, which was $15.7 \mathrm{~cm}^{2}$. Cylindrical shape with a diameter of $10 \mathrm{~mm}$ and length of $50 \mathrm{~mm}$, a beam with a side length of $7.85 \mathrm{~mm}$ and height of 50 $\mathrm{mm}$, and a cone with a diameter of $20 \mathrm{~mm}$ and length of $48.98 \mathrm{~mm}$.

\subsection{Specimen Preparation}

For specimen preparation, a hole with a diameter of $3 \mathrm{~mm}$ was made at one end of the specimen. Then it was connected to the Support Rod. Supporting rods must be electric insulators in all parts exposed to the solution. Then at both ends of the specimen coated using gun glue. Before dipping into the solution, the sample was washed with water followed by alcohol. The specimen was then dried and weighed to $0.1 \mathrm{mg}$. 
MATERIALS RESEARCH COMMUNICATIONS 1(1) (2020) 8-16

Tubagus et. al.

\subsection{Procedure method of DnVGL-RPB401}

Sacrificial anode and steel cathode specimens were connected to the positive and negative poles respectively with a DC constant current source. Then the current flowing through the circuit must be adjusted to produce anodic current density based on the initial surface area of the specimen exposed to the solution, that was the first day was $1,5 \mathrm{~mA} / \mathrm{cm}^{2}$, the second day was $0,5 \mathrm{~mA} / \mathrm{cm}^{2}$, the third day was $4,0 \mathrm{~mA} / \mathrm{cm}^{2}$, and the fourth day was 1,5 $\mathrm{mA} / \mathrm{cm}^{2}$. Current density must be controlled with an accuracy of up to $0,1 \mathrm{~mA} / \mathrm{cm}^{2}$ and must be maintained throughout the period. The total time was $96 \pm 4$ hours. At the end of each, the anode potential was measured at three node positions. Specimens should not be cleaned of corrosion products before measurement. Then to calculate the electrochemical efficiency using the following formula:

$$
e=\frac{C \times 1000}{W}
$$

Where $e$ is the electrochemical anode(ampere-hour $/ \mathrm{Kg}$ ), $C$ is the total change in current (ampere.hour), $W$ is the weight of the anode lost (grams), then to calculate the anode efficiency is as follows:

$$
\eta=\frac{e}{2976,256} \times 100 \%
$$

Where $\eta$ is the efficiency of the sacrificial anode, and 2976,256 is the capacity of pure Al Anode (A-hr / Kg)

\subsection{Salinity Calculation}

The salinity calculation by evaporation of 1 liter of Kenjeran seawater, then calculated the salinity with the equation

In this research, salinity of Kenjeran seawater was $3.04 \%$

$$
\text { Salinity }(\%)=\frac{\text { Dissolved mass }}{\text { total mass }}
$$

\subsection{Electrolyte Preparation}

To make 10 L substitute sea water with $3.04 \%$ salinity was to use ASTM D1141, which dissolves pure 304 grams of $\mathrm{NaCl}$ into 8 liters of distilled water. Then thaw it up to 10 liters. So that it will produce a pure $\mathrm{NaCl}$ solution with $3.04 \%$ salinity. For a solution of kitchen salt, dissolve 323 salt in 8 liters of distilled water. Then thaw it up to 10 liters, resulting in dissolved salt salt with $3.04 \%$ salinity.

\section{Result and Discuss}

In the DnVGL-RPB401 method, there were 2 times the potential anode sacrificial tested, that was Closed Circuit Potential (CCP) and Open Circuit Potential (OCP)

\subsection{OCP with Variation of Anode Shape}

Open Circuit Potential (OCP) is the potential value of the anode before it is connected to the material to be protected. The following Figure 1 represents the OCP value of the anode variation in the sacrificial anode shape with the same medium pure $\mathrm{NaCl}$ solution in the DnVGL-RPB401 method. Variations in the shape of the anode in the form of cones, beam and cylinders with the same surface area of $15.7 \mathrm{~cm}^{2}$ 
MATERIALS RESEARCH COMMUNICATIONS 1(1) (2020) 8-16

Tubagus et. al.



Fig. 1. Anode OCP value with variation of anode shape.

The most negative open circuit potential value occurs in the beam anode form $-1025 \mathrm{mV}$, cylindrical anode form $-985 \mathrm{mV}$, and the most positive OCP value occurs in the cone anode shape $-928 \mathrm{mV}$.

\subsection{OCP with Variation of Electrolyte}

The following Figure 2 represents the OCP value of anode variations in electrolyte with the same sacrificial anode form, which was the cylinder in the DnVGL-RPB401 method. Electrolyte variations in the pure $\mathrm{NaCl}$ solution, a solution of kitchen salt and seawater. The three electrolyte media in this research had $3.04 \%$ salinity.

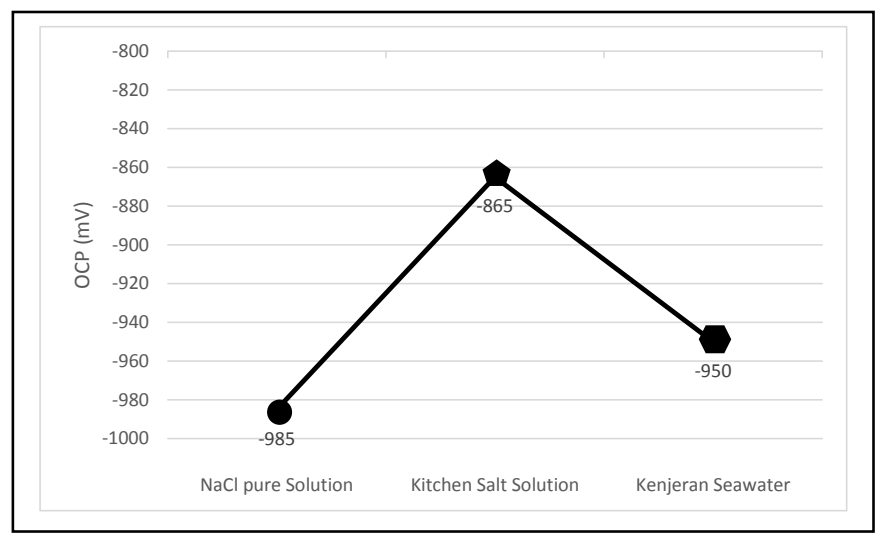

Fig. 2. Anode OCP value with variation of electrolyte.

In Figure 2 shows the OCP value of the sacrificial anode variation of electrolyte. From Figure 2 it can be seen that the most negative OCP value occurs in pure $\mathrm{NaCl}$ solution that was equal to $-985 \mathrm{mV}$, then in seawater of $-950 \mathrm{mV}$ and in kitchen salt solution of $-865 \mathrm{mV}$. This is due to differences in the composition of the electrolyte.

In general, corrosion in seawater or seawater environments is caused by an abundance of chloride ions $\left(\mathrm{Cl}^{-}\right)$, for example Sodium Chloride $(\mathrm{NaCl})$. Because chloride ions themselves reduce the resistance of the aluminum film layer and help release the aluminum film layer to water. in addition, seawater also contains sulfate ions $\left(\mathrm{SO}_{4}{ }^{2-}\right)$ which increase the depth of local corrosion (Pitting) and inhibit the formation of the oxide layer (Vargel, 2004). In the Kitchen Salt Solution there is Potassium Iodide, Potassium Iodide itself acts as a corrosion inhibitor in low carbon steel (Khadom, 2018). The following was Table 2 results of the composition electrolyte with a salinity of $3.04 \%$. 
MATERIALS RESEARCH COMMUNICATIONS 1(1) (2020) 8-16

Tubagus et. al.

Table 2. Composition of electrolyte

\begin{tabular}{|c|c|c|c|}
\hline Elektrolit & $\mathrm{NaCl}(\mathrm{mg} / \mathrm{l})$ & $\mathrm{Na}_{2} \mathrm{SO}_{4}(\mathrm{mg} / \mathrm{l})$ & $\mathrm{KI}(\mathrm{mg} / \mathrm{l})$ \\
\hline Air Laut Kenjeran & 261000 & 38.60 & 8.60 \\
\hline Larutan Garam Dapur & 256000 & 29.10 & 42.60 \\
\hline Larutan $\mathrm{NaCl}$ murni & 283000 & 38.60 & 82.50 \\
\hline
\end{tabular}

The highest $\mathrm{NaCl}$ content occurs in electrolyte of pure $\mathrm{NaCl}$ solution, so that in electrolyte the pure $\mathrm{NaCl}$ solution of the anode metal has the most negative OCP value, and causes the $\mathrm{NaCl}$ solution to be more easily subjected to electric transfer (Widodo, 2018). then the second most is sea water kenjeran. Whereas electrolyte in kitchen salt solution had the most positive OCP, this was due to the lowest $\mathrm{NaCl}$ content and the presence of potassium iodide in kitchen salt solution, because the presence of potassium iodide in aluminum accelerated the growth of the oxide layer on aluminum metal, making metals more difficult to undergo electron transfer (ElMaghraby, 2009).

\subsection{CCP with Variation of Anode Shape}

Closed Circuit Potential (CCP) is the potential value after the anode is connected to the material to be protected. CCP values in this method are CCP values for 4 days, then averaged. Following Figure 3 was the CCP value on the DnVGL-RPB401 Method

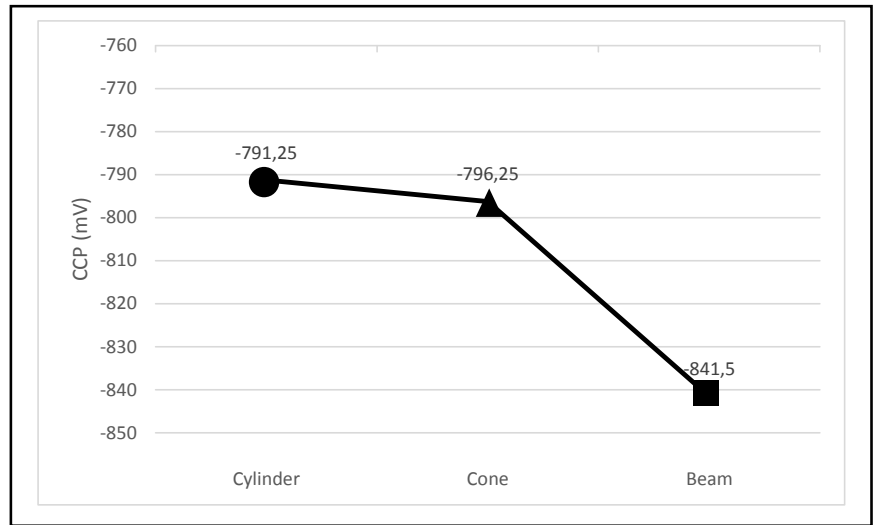

Fig. 3. Anode CCP value with variation of anode shape.

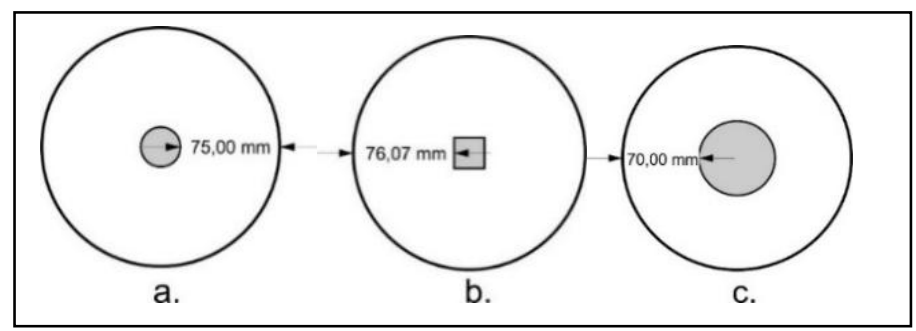

Fig. 4. Illustration of geometry distance between anode and cathode with anode Shape a) cylinder anode shape, b) beam anode shape, c) cone anode shape.

In Figure 3 can be seen that the most negative Closed Circuit Potential value occurs in the beam $-841.5 \mathrm{mV}$, the $-796.25 \mathrm{mV}$, and $-791.25 \mathrm{mV}$ Cylinder. this is because the distance between the anode and the cathode is different. The farther the distance between the anode and the cathode, the more negative the anode potential is. so that the most negative electrical potential occurs in the beam form, because the farther the distance between the anode and the cathode the more negative the electrical potential (Mujezinovic, 2016). 
MATERIALS RESEARCH COMMUNICATIONS 1(1) (2020) 8-16

Tubagus et. al.

In Figure 4 was an illustration of the geometry of the distance between the anode and the cathode. the farthest distance between the anode and the cathode occurs in the beam anode shape, this was caused the electric potential in the most negative anode form. While the electrical potential in the cylinder shape was more positive than the beam shape because the distance between the anode and the cathode was closer than that of the beam form.

\subsection{CCP with variation of Electrolyte}

Following Figure 5 was the CCP value on the DnVGL-RPB401 (2017) Method. This CCP value indicates whether or not a material was protected.

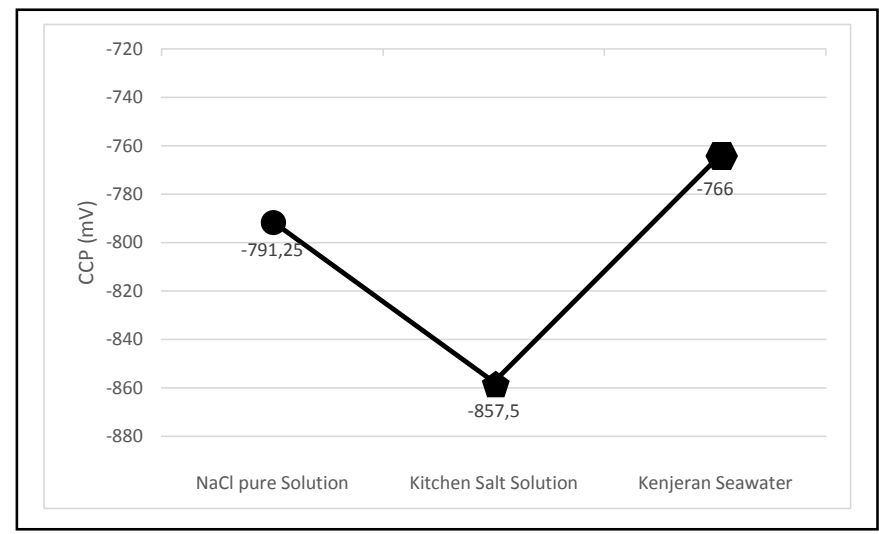

Fig. 5. Anode CCP value with variation of electrolyte.

From Figure 5, it can be seen that the most negative CCP value occurs in kitchen salt solution which was $857.5 \mathrm{mV}$, then pure $\mathrm{NaCl}$ solution was $791.25 \mathrm{mV}$, and the one that has the most positive potential occurs in the salted sea water solution which was $-766 \mathrm{mV}$.In the variation of electrolyte, CCP value is influenced based on the composition level in the electrolyte. The levels of electrolyte composition can be seen in Table 2

From the $\mathrm{CCP}$ values on these three electrolyte. Can be seen in the electrolyte of kitchen salt solution, the anode metal has the most negative CCP value and makes the anode metal less corrosive, this is because the $\mathrm{NaCl}$ and $\mathrm{NaSO} 4$ content is the least and there is potassium iodide. Because $\mathrm{NaCl}$ and $\mathrm{NaSO} 4$ itself is one of the causes of corrosion in metals (Mujezinovic, 2016) and the presence of potassium iodide which is one of the inhibitors of corrosion in metals (Vargel, 2004). while the most positive CCP value occurs in the medium of seawater electrolyte, this is due to more $\mathrm{NaCl}$ and $\mathrm{Na} 2 \mathrm{SO} 4$ content than electrolyte in kitchen salt solution and has the least KI among the three electrolyte.

\subsection{Anode efficiency with Variation of Anode Shape}

After got the anode mass lost during tested. Then calculated the anode current capacity with equation 1, then compare the anode current capacity with the theoretical Al-pure anode current capacity to got the anode efficiency. Following Figure 6 was the efficiency value of the sacrificial anode in the DnVGL-RPB401 method. 
MATERIALS RESEARCH COMMUNICATIONS 1(1) (2020) 8-16

Tubagus et. al.

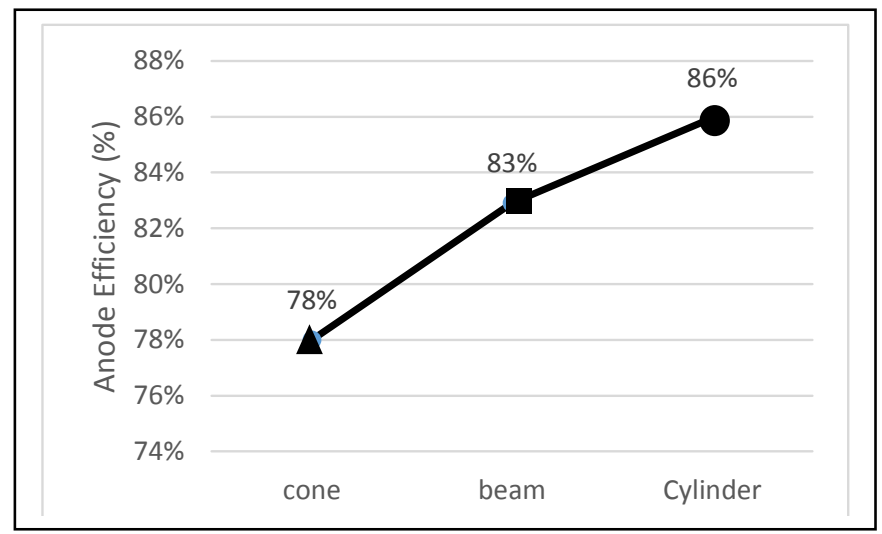

Fig. 6. Anode efficiency with variation of anode shape.

In Figure 6, the highest anode efficiency was $86 \%$ that was cylindrical anode efficiency, $83 \%$ beam shape and $78 \%$ cone shape. This was because the distance between the anode and the cathode is different. Resulting in different current density distribution. The closer the distance between the anode and the cathode the higher the current density. And the higher the current density the easier it is for metals to dissolve (Turkuvic, 2017).

From Figure 7 it can be seen that in the form of a cylindrical anode, current densities are spread evenly. In the Beam anode form, the distribution of current density on the horizontal axis is not evenly distributed. Whereas in the form of cone anodes, the distribution of current density is not evenly distributed on the vertical axis.

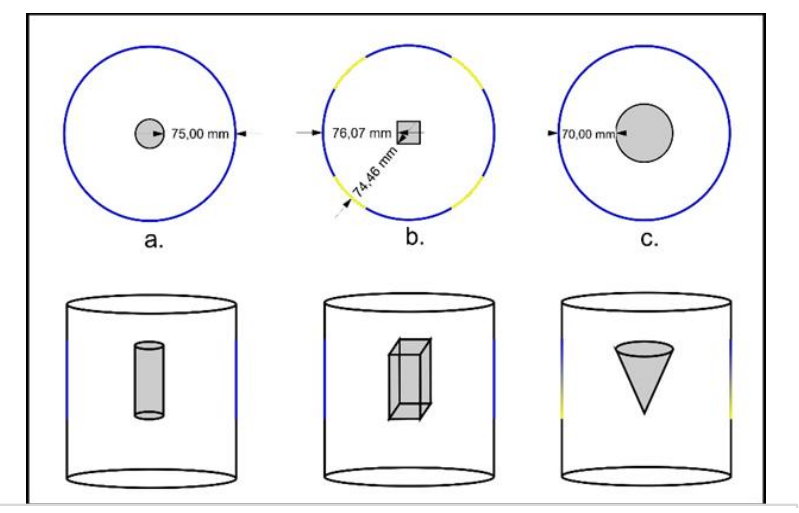

Fig. 7. Illustration of distribution of current meeting distribution in DnVGL-RPB401 method with anode form a) cylinder b) beam c) cone.

From Figure 7, it can be seen that part b, that was the shape of the beam has four corner points which have a distance between the anode and the cathode that is closer than the other side. The highest current density at the surface of the anode occurs at the surface of the anode closest to the cathode, the distribution of current density is inversely proportional to the surface of the anode, ie the galvanized surface of the anode will dissolve over time, so that the surface of the anode closest to the cathode will be more soluble (Turkuvic, 2017). So that at these four corner points flowing denser currents are higher than the other side. This causes the four corner points on the surface of the anode to dissolve more easily as Figure 8.

From Figure 7 Section c, we can see that in the shape of the cone anode on the vertical axis there are regions that have different distances between the anode and cathode, ie the lower the cone side the farther the distance between the anode and the cathode. This makes the current density that flows on the surface of the anode different. And the anode area closest to the cathode will flow the highest current density. This causes the anode to dissolve more easily. And in Figure 9 it can be seen that the conical sacrificial anode at a point closer to the cathode is more soluble. 
MATERIALS RESEARCH COMMUNICATIONS 1(1) (2020) 8-16

Tubagus et. al.

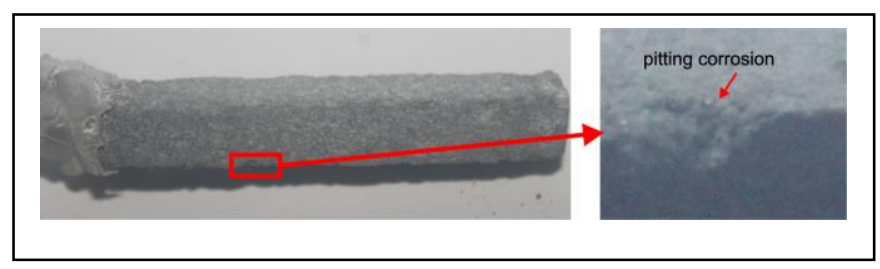

Fig. 8. Beam specimens after DnVGL-RPB401 method.

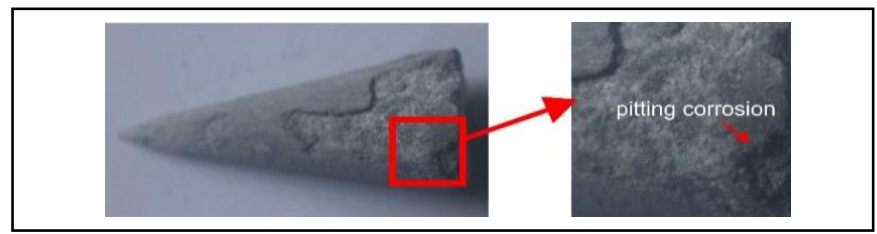

Fig. 9. Cone specimens after DnVGL-RPB401 method.

While from Figure 7 part a, that the current density flowing in the cylindrical shape is spread evenly on the surface of the anode both horizontally and vertically, this is because the distance of the anode to the cathode in the cylinder shape has the same distance. So it can be seen in Figure 10 that in the cylindrical shape there are no areas that experience faster dissolution.



Fig. 10. Cylinder specimens after DnVGL-RPB401 method.

\subsection{Anode Efficiency with variation of electrolyte}

After got the anode mass lost during tested. Then calculated the anode current capacity with equation 1 , then compare the anode current capacity with the theoretical Al-pure anode current capacity to got the Anode efficiency. Following Figure 11 was the efficiency value of the sacrificial anode in the DnVGL-RPB401 method.

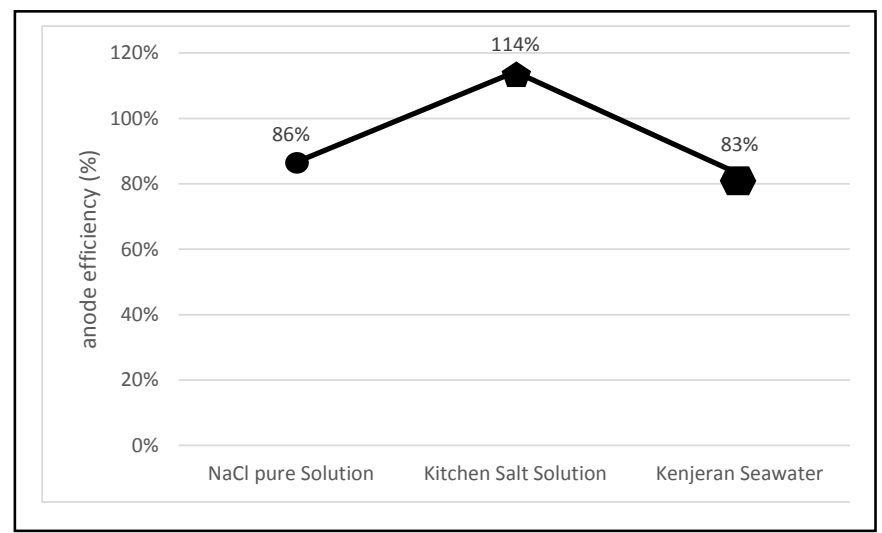

Fig. 6. Anode efficiency with variation of anode shape. 
MATERIALS RESEARCH COMMUNICATIONS 1(1) (2020) 8-16

Tubagus et. al.

In the form of a cylindrical anode with 3 different electrolyte. That was the electrolyte of pure $\mathrm{NaCl}$ solution, a solution of salt and Kenjeran seawater. From Figure 11 it can be seen that the highest efficiency is a sacrificial anode with an electrolyte media of salt solution of $114 \%$, then pure $\mathrm{NaCl}$ solution of $86 \%$, and the smallest efficiency is the medium of seawater which is $83 \%$.

In the DnVGL-RPB401 method, the sacrificial anode which experienced the most mass reduction occurred in the medium of seawater water, this was due to the $\mathrm{NaCl}$ levels in table 2 which were higher than the kitchen salt solution, which was $261000 \mathrm{mg} / \mathrm{l}$ and the least potassium iodide level was among the three electrolyte media, which is $8,6 \mathrm{mg} / \mathrm{l}$. So that the most experienced mass reduction in pure $\mathrm{NaCl}$ has a lot of $\mathrm{NaCl}$ levels, which is $283000 \mathrm{mg} / \mathrm{l}$, and has the most $\mathrm{KI}$, which is $82,5 \mathrm{mg} / \mathrm{l}$. This results in a greater reduction in anode mass compared to flat sea water but less than kitchen salt solution. And the level of sodium sulfate (Na2SO4) for the media of pure nacl solution and sea water kenjeran is the same, which is equal to $38,60 \mathrm{mg} / \mathrm{l}$.

In kitchen salt solution, it has the lowest $\mathrm{NaCl}$ level, which is 2,56 grams, has the least $\mathrm{Na}_{2} \mathrm{SO}_{4}$ level, which is $29,10 \mathrm{mg} / \mathrm{l}$, plus the presence of $\mathrm{KI}$ of $42,60 \mathrm{mg} / \mathrm{l}$. This results in the least amount of lost anode mass and has the greatest anode efficiency compared to the three electrolyte.

\section{Conclusions}

Based on the research conducted it can be concluded that:

a) In the anode shape variation, the highest anode efficiency occurs in the cylindrical anode shape of $86 \%$, quadrilateral prism shape $83 \%$ and conical shape $78 \%$.

b) In the electrolyte media variation, the highest anode efficiency occurs in the media of kitchen salt solution that is $114 \%, 86 \%$ pure $\mathrm{NaCl}$ solution and $83 \%$ absolute sea water.

\section{Acknowledgments}

The author would like to thank Mr. Basuki and Mr. Tari as Tendik in the material engineering department who have assisted in preparing our specimens, and to Mr. Ifthar Muhammad Ghazy who has helped in preparing the tools and materials in this study, so that this research can be completed.

\section{References}

DNVGL. (2017), DNVGL-RPB401 Recomendation Practice Cathodic Protection design. DNV GL, AS

El-Maghraby, A. A. (2009), 'Corrosion Inhibition of Aluminum in Hydrochloric Acid Solution Using Potassium Iodate inhibitor', The Open Corrosion Journal, Vol 2 pp. 189-196

Khadom, Anees A. (2018), 'Potassium Iodide as a Corrosion Inhibitor of Mild Steel in Hydrochloric Acid : Kinetics and Mathematical Studies', Journal of Bio- and TrilboCorrosion 4:17

Mujezinovic, Adnan. (2016), 'Modeling of the Galvanic Anoda Cathodic Protection System with Dynamic Polarization Characteristics', International Journal of Chemistry and Chemical Engineering Systems, Vol 1, pp. $96-100$

Mujezinovic,Adnan and Irfan Turkovic. (2017), 'Review On Cathodic Protection System Modelling', Review Scientific Paper, Vol 11, pp. 81-91

Roberge, Pierre R. (2008), Corrosion Engineering Principles and Practice, McGraw-Hill, New York, USA

Vargel Cristian, Michael Jacques and Dr. Martin P.Schmidt. (2004), Corrosion of Aluminium, Elsevier Ltd, Netherlands.

Widodo, Chomsin Sulistya. (2018). 'The Effect of $\mathrm{NaCl}$ Concentration on the Ionic $\mathrm{NaCl}$ Solutions Electrical Impedance Value using Electrochemical Impedance Spectroscopy Methods'. AIP Conference Proceedings. 17 Oktober 2018 